\title{
DRAMMATURGHI SI NACE O SI DIVENTA?
}

\author{
ARE DRAMATISTS BORN OR MADE?
}

Patrizia Monaco

Autrice teatrale

\section{RIASSUNTO:}

Con il sottotitolo: Esperienza decennale nell'insegnamento della drammaturgia ad allievi dai 7 ai 70 anni, sintetizzo un mio esperimento. Il Comune di Santa Margherita Ligure sponsorizza un corso nel quale gli allievi, d'ogni età, cultura, mestiere e professione, scrivono assieme un copione che verrà interpretato da loro stessi, seguendo il "Decalogo" da me scritto, con molta immodestia.

\section{AbStRACT:}

With the subtitle: Ten years' experience ofteaching drama to pupils aged 7-70, I give a brief account of my teaching experience. The Comune of Santa Margherita Ligure sponsors a course in which participants of all ages, and different cultural, social and work backgrounds, collaborate in writing a script which they will perform themselves, in accordance with my immodestly entitled "Decalogue".

\section{KEYwORDS:}

sintesi, Dialogue, characters, concision, involvement.
Dialogo, pers
immedesimazione.

personaggio, 
In questa comunicazione dal tono familiare intendo parlare della mia esperienza nell' insegnamento della drammaturgia che svolgo da più di dieci anni in diversi istituti scolastici, università italiane e straniere biblioteche, scuole di recitazione ed inoltre all'Istituto di Cultura Italiano ad Istanbul.

Dopo una breve introduzione, mi propongo di illustrare le diverse modalità con cui insegno la scrittura per la scena. Più tecnica e professionale per aspiranti attori e studenti di teatro, più semplice e giocosa per i bimbi delle scuole elementari. Mi soffermerò quindi su un esperimento che svolgo a Santa Margherita Ligure, Genova, dove il Comune sponsorizza un corso gratuito per la popolazione. In questo corso, seguendo una mia metodologia, gli allievi, dì ogni età, cultura, mestiere o professione, scrivono parti di un unico testo che poi verrà interpretato da loro stessi. Si tratta non solo di insegnare drammaturgia e recitazione, ma si dimostra concretamente quali debbano essere le caratteristiche di un testo affinché sia adatto alla scena. La tecnica si riassume in un "Decalogo" da me scritto e così definito, con molta immodestia. Per caso, quasi per caso sono diventata insegnante di drammaturgia e il fatto ora mi appassiona così tanto che ho pensato valesse la pena di doverne scrivere. Prima però mi vorrei presentare. Sono autrice teatrale, con qualche incursione nella radio e nel cinema, e i miei testi sono stati premiati, rappresentati e pubblicati in Italia e all'estero. E' la mia professione.

\section{DRAMMATURGIA}

Sono laureata in storia del teatro, $\mathrm{l}^{\prime}$ ho fatto per disciplinare la mia cultura teatrale ma non ho mai pensato di intraprendere la carriera accademica. Non ne posseggo le doti. Senza andare lontano, osservando il lavoro che hanno fatto sui miei lavori la professoressa Milagro Martin Clavijo e il prof Roberto Trovato, la ricerca meticolosa, la profondità, il porsi onestamente e criticamente di fronte ad un'opera, l'umiltà, insomma, loro non inventano niente. Io invento tutto!!! Fantasia sfrenata. Ma se dall'insegnamento ne sono uscita dalla porta, poiché in fondo non ne ero mai entrata, ecco che sono rientrata dalla finestra e qualche anno fa ho insegnato drammaturgia all'Università, prima a Dublino, dove avevo lavorato come lettrice di italiano anni prima e poi a Genova, invitata dal prof Trovato. Insegnare drammaturgia all'Università è peraltro privilegiato, gli studenti di storia del teatro o addirittura del corso di drammaturgia, conoscono la struttura di un testo teatrale, ne hanno letti e analizzati, ma soprattutto, sanno, cos'è il teatro, o come è fatto un edificio teatrale. Insegnare loro a scrivere un testo non è in fondo troppo arduo. Bastano le poche ma necessarie regole che disciplinano la drammaturgia, "The Art of Playwriting", e il gioco è fatto. Loro studiano Shakespeare e Goldoni, molto probabilmente non scriveranno mai come Shakespeare o Goldoni ma purtuttavia imbastiranno un qualcosa che possa assomigliare ad un testo teatrale. 
Ma per chi a teatro non c'è mai stato? I bambini e i ragazzi ad esempio. Ed ora arriviamo al caso. Ad una svolta drammatica della mia vita mi ritrovai a vivere in quella che era la nostra seconda casa, in Riviera, ed ecco aprirsi una prospettiva mai neppure pensata, insegnare a scrivere di teatro. Prima del mio incontro ravvicinato con una coppia di tumori, facevo allegramente la spola fra Roma e Parigi a organizzare spettacoli miei e altrui, emissaria del Sindacato Autori, dell'Associazione Autori e quanto altro. Chi vive nella Riviera Ligure anche se Genova non è lontana, non ha molte opportunità di andare a teatro e per i ragazzi del Millennium - nati negli anni 2000 ormai i video sono l'unica forma di comunicazione e intrattenimento, e su schermi sempre più ristretti, altro che le tavole di un palcoscenico. Ad un amica attrice, regista e insegnante di recitazione, Patrizia Ercole, che teneva un corso di recitazione nelle scuole Medie (all'inizio degli anni duemila era possibile nelle scuole italiane mettere a disposizione parte dei fondi per fare teatro. Ora non hanno più neanche i soldi per la carta igienica... ) era stato chiesto di mettere in scena i "Promessi Sposi" , di Alessandro Manzoni, nella riduzione per ragazzi di Roberto Piumini.

Chiese il mio aiuto e fui arruolata anch' io. Per fare in modo che riscrivessero i Promessi Sposi pensai alla mia esperienza di autrice, alle mie letture, comprai libri su "The Art of Playwriting" - quasi tutti in inglese - e per loro stilai il mio decalogo.

\section{DECALOGO DI DRAMMATURGIA}

Per scrivere un testo che deve essere rappresentato, bisogna ricordare queste semplici regole che io, con molta immodestia, ho chiamato "Il decalogo". Il testo scritto per il teatro, la cui particolarità è di creare personaggi che saranno poi interpretati da attori, su uno spazio delimitato, è formato dai seguenti elementi:

1. DIALOGO, composto dal linguaggio e dalle pause e dai silenzi, altrettanto carichi di significato delle parole. Il dialogo deve sembrare naturale senza avere del linguaggio reale la sciatteria e la debolezza. Deve essere ricco, sintetico e soprattutto, deve portare avanti l'azione. Il linguaggio è composto di PAROLE scritte non per essere lette bensì per essere dette;

2. DIDASCALIE, che descrivono l'azione e gli stati d'animo dei personaggi;

3. CARATTERIZZAZIONE dei personaggi. Ognuno parla e si muove in modo diverso, proprio come gli esseri umani. I personaggi che dovranno sul palco, "stare in piedi da soli", saranno tridimensionali, non solo bocche che siano il portavoce delle idee dell'autore;

4. RITMO, che deve variare di continuo, come nella musica. Si scrive pertanto ad alta voce;

5. SCENOGRAFIA VERBALE, cioè evocare i luoghi con le parole; 
6. SOTTOTESTO, ciò che si nasconde al di là delle parole;

7. CONFLITTO, in ogni scena, non necessariamente violento, ma una contrapposizione, anche pacata, di idee, decisioni e sentimenti;

8. ETERNO PRESENTE, poiché sulla scena tutto avviene qui ed ora;

9. SOSPENSIONE DELL'INCREDULITA', riuscire a far credere allo spettatore tutto quanto si svolge sulla scena;

10. IL PUBBLICO, la cui attenzione deve essere catturata sin dalle prime battute, ma la cui curiosità sarà soddisfatta solo alla fine.

“Un irresistibile impulso alla metamorfosi di se stesso e ad agire con altri corpi e altre anime... è questa la prima condizione di ogni arte drammatica, solo chi prova questo impulso e questo incantesimo è drammaturgo" . Così scriveva Nietzsche, e così mi sento io quando scrivo e lo stesso deve essere per i miei allievi!!!

A corollario, facevo esempi con la stesura di un romanzo, in cui si può scrivere di quel che vede o pensa un personaggio, mentre in un copione tutto deve essere risolto con dialogo e gesti. Ribadire inoltre la regola aurea: non spiegare, mostra. Non potendo dire che un personaggio è triste o arrabbiato, bisogna mostrarlo attraverso le sue parole, la sua postura, le sue azioni, l'espressione del volto. In questo aiutano le didascalie, che servono però solo all'attore e al regista, dirette quindi a loro e non al pubblico, che non può accedere a molte informazioni, come accade invece al lettore di un romanzo o di un racconto.

Non fu facile, ma neanche troppo difficile, in fondo c'era un testo e che testo, il capolavoro della letteratura italiana, e ne nacque uno spettacolo vivace e divertente. I ragazzi più disciplinati vollero scrivere e interpretare la parte dei "bravi" cioè quella dei cattivi e si dimostra come il teatro sia una ottima terapia per canalizzare le pulsioni più nascoste.

Poi vennero i bambini delle elementari, in Italia dai 6 ai 10 anni. La loro insegnante voleva mettere in scena un Pinocchio multietnico, basandosi sul fatto che esistono molte versioni di Pinocchio in ogni parte del mondo. Non traduzioni, versioni diverse. Chiamo questa altra amica: " Pinocchiologa".

Orbene, questi bambini non sapevano cosa è il teatro. Videogiochi, playstation, computer, cellulari ok, ma il teatro? Lo chiesi la prima mattina, davanti a file di banchi occupati da bimbi di ogni colore, con le faccine più annoiate che curiose.

"Cosa è il teatro?" Dopo varie espressioni perplesse, neanche avessi chiesto di descrivere un marziano, e diversi “Boh!” raccolsi due risposte folgoranti. 
“E' la grande casa dove fanno i cartoni animati dal vivo ".

"Dove si piange". Detto da un bambino siciliano che poverino lui era stato trascinato a vedere le tragedie greche a Siracusa. A queste simpatiche bestioline riuscii ad insegnare a scrivere una loro sceneggiatura e poi ad interpretarla. Anche in questa occasione, usai in maniera soft il decalogo, non si può citare troppo Aristotele e la formula: "a teatro è da preferirsi l'impossibile verosimile al possibile incredibile" ma il concetto, "qui ed ora" , viene compreso per intuito, dopo le prime resistenze.

Il teatro è una magia che li cattura, specialmente se sono loro stessi a farlo. Fu un successone e questo mi portò alla considerazione che tutti possono scrivere per il teatro, perché tutti dentro possiedono un tesoro. Bisogna solo farlo uscire e mostrarlo con le tecniche adatte. Sfruttammo i pregiudizi che i bimbi senza accorgersene e senza cattiveria ripetono perché sentiti dai loro genitori o per strada. Questi pregiudizi, inglobati nella storia, diedero un pizzico di sale in più. I genitori e gli adulti del pubblico quasi se ne scandalizzarono, poi ne risero, e col ribaltarsi dei clichès, vi fu una catarsi. Come quando viene rubata la torta di Pinocchio e subito si pensa ai pinocchi rumeni, perché, si sa, sono zingari e ladri. Mentre ad un certo momento bisogna distrarre qualcuno e si pensa di stordirli con aranciata all'erba" e allora si ricorre ai pinocchi colombiani perché, si sa, loro spacciano. Ovviamente né i pinocchi rumeni né quelli colombiani sapevano fare quello di cui erano accusati. La storia verteva sul compleanno di Pinocchio, cioè l'anniversario della prima pubblicazione del libro di Collodi, e a questa gran festa vengono invitati i pinocchi di tutto il mondo. Al bambino russo, col suo mediatore culturale accanto, si chiese di portare il suo contributo e ci piacque che lui ripetesse la parola "bolshoi", grande, davanti alla torta. Un richiamo, per noi adulti, al prestigioso tempio del balletto. Ognuno potè infilare qualche parola della sua lingua o del suo dialetto e questa babele contribuì alla vicacità del compleanno del burattino di legno, festa che all'inizio è funestata dalla scomparsa della torta e dalla mancanza della madrina, la lumaca, che anche se si era messa in viaggio mesi prima era comunque in ritardo. La lumaca era una bambina avvolta in una sagoma a forma della chiocciola del web. Ci arrivammo assieme, a questa trovata. E si celebrò così l'elogio della lentezza. Riuscii a far veicolare quanto a me preme di più nella società contemporanea: la lotta contro il razzismo, la paura del diverso e un contrastare l'ansia e la fretta che ci pervadono. I bambini risposero con entusiasmo a queste istanze e furono complici e partecipi. Io davo le direttive, assieme si improvvisava in classe e poi insegnanti e mediatori li aiutavano a mettere per scritto quanto detto. Infine, io rivedevo il tutto e mostravo loro come le parole devono essere scritte per essere dette e non per essere lette. Decalogo docet.

Poi ci furono due anni in una scuola professionale, dove fui assunta perché alle ragazze del corso di costumistica era stato chiesto di preparare uno spettacolo sulla tela 
di Genova, cioè il jeans (o toile de Genes) sponsorizzato da un fabbricante di pantaloni che ne avrebbe steso un paio della lunghezza di trenta metri al Porto Antico di Genova.

Non facile. Ma ci riuscimmo. Sempre grazie al mio decalogo, letto solo in seguito, quando entrai nelle loro simpatie perché le feci dapprima "scrivere" usando i cellulari. Poiché loro si parlavano quasi esclusivamente attraverso il cellulari, e in seguito hanno capito che si può fare anche senza!!!

Una sola di loro, su diciotto nella classe, era stata a teatro, pertanto dovetti prendere esempi di come appare una scena e di come la si interpreta da film visti in televisione. E pensare che stavano a Genova, una città che vanta una dei migliori teatri stabili d'Italia.

Il punto di partenza fu: col cellulare si può mentire su dove ci si trova, ma se presi da entusiasmo ci facciamo un selfie con lo sfondo, la verità viene a galla. Trovata l'idea, improvvisare e poi scrivere una trama d'amore e piccoli tradimenti il tutto "avvolto nella tela di Genova “divenne via via sempre più avvincente.

Insegnare drammaturgia fu piuttosto agevole a Istanbul presso l'Istituto Italiano di Cultura, frequentato da persone colte, italiane e turche, grandi lettori e frequentatori di teatro - molto attiva e seguita la scena teatrale, prima che vi fossero gli ultimi tragici attentati terroristici, - ma con loro non si trattava di mettere in scena quello che scrivevano. Pertanto furono esercitazioni piuttosto buone.

Con loro potei approfondire i concetti, "a teatro tutto è finto ma nulla è falso" e il dialogo che "non è reale, ma sembra reale". Lo stesso era accaduto agli studenti del Corso di drammaturgia del prof Trovato, poiché oltre ad impartire lezioni tratte dal decalogo mi divertii a tracciare sulla lavagna alcuni disegni per illustrare la struttura del testo teatrale. Il quale deve, è lapalissiano, avere un inizio, climax, e finale, avere trama e possibile sottotrama, essere gravido di sottotesto e auspicabili colpi di scena. I disegni prendevano quindi curiosamente la forma di un drago con le relative creste. Piacevole parlare con alcuni di loro, che avevano letto e apprezzato testi classici e moderni, frequentato assiduamente anche i vari teatrini sperimentali di Genova e dintorni e mordevano il freno per cimentarsi loro stessi. Semai non fu facile vincere le loro resistenze a correggersi, a capire che una battuta deve essere breve, insomma, non compiacersi troppo dei bei discorsi. Solo leggendoli e interpretandoli concretamente nella classe, spostando gli scomodi banchi, in piedi, riuscii a far comprendere loro quanto il teatro debba essere dinamico, che anche quando si scrive, si scrive in piedi. Si debbono dire ad alta voce le battute ed entrare con tutto il peso del nostro corpo in ogni personaggio, rileggendo quanto appena scritto camminando per la stanza. Stare attenti che se un personaggio si abbandona ad uno sproloquio, l'altro attore che fa nel frattempo? 
Feci molti esempi di sticomitia, le battute a raffica che si rincorrono l'un l'altra usando le stesse parole con effetto staffetta, da Goldoni a Pinter, e alla fine con grande sofferenza, rinunciarono ai paroloni, barattando la magniloquenza per una sintetica teatralità. "La sintesi è la sorella del talento" , diceva Cechov. Frase che consigliai loro di scrivere su un post-it e applicarla allo schermo del computer.

Nell'aula magna, alla fine del corso, ci esibimmo in un eccellente reading, con copioni di grande intensità, alcuni molto divertenti, recitati dagli studenti stessi, dalla sottoscritta e dall'ineffabile Roberto Trovato. In alcuni si scavò nei rapporti di coppia, in altri nel disagio giovanile, altri furono metateatrali, tutti di ottimo livello.

L'uso del decalogo e dei suoi corollari, nonché queste ultime considerazioni sulla persistenza della letterarietà e la susseguente quindi necessità di ribadire il concetto che si scrive come si recita, in piedi, anche metaforicamente, sono la base del corso su cui intendo soffermarmi per la sua peculiarità.

Il corso di "Drammaturgia e Recitazione", che occupa gran parte della stagione invernale, da novembre a febbraio è quello che svolgo in un piccolo teatrino, i primi tempi in biblioteca, nel Comune di Santa Margherita Ligure, cittadina a meno di trenta chilometri da Genova.

Un corso diverso dagli altri, per durata, più breve, e per tipologia. Gli altri duravano un intero anno scolastico o accademico, e avevano allievi di tipologia uniforme. Studenti universitari a Genova e Dublino, bambini e ragazzi, studentesse costumiste, attori in scuole di recitazione, e prevalentemente insegnanti a Istanbul.

Il corso di drammaturgia e recitazione, gratuito per la popolazione, raccoglie persone d'ogni età, provenienza, professione e mestiere. Le classi spesso superano le 25 presenze. Giovani e anziani, due anni fa si andava dai 7 ai 75 anni, pensionati e disoccupati, studenti e insegnanti, architetti postini e poliziotti, sottufficiali di Marina e casalinghe, tecnici cuochi ed pasticceri, elettricisti, spazzini, operatori nel sociale. badanti e giornalisti. Liguri doc, italiani d'ogni regione e stranieri dall'Ucraina, Francia, Romania, Tunisia, Brasile.

Un cocktail dai sapori bene assortiti o una miscela esplosiva? L'una e l'altro. Pertanto io io mi ritrovo spesso a comportarmi come un misto fra una maestra d'asilo e una domatrice di tigri. Ah, gli aspiranti scrittori... che caratterini, e che pretese!!! Dai loro scritti, spesso a mano, o elaborati sul computer con impaginazioni improbabili, ebbi la conferma che tutti possono scrivere, perché tutti hanno qualcosa da dire, perché come avevo già scoperto coi bimbi, tutti hanno un tesoro dentro di loro. Basta saperlo cercare, basta avere pazienza, e... basta ascoltarmi... 
“Scrivere significare raggiungere l'ignoto dentro di noi " affermava Marguerite Duras e la maggioranza dei miei allievi ha intrapreso negli anni questa affascinante avventura.

Poiché svolgo un lavoro creativo professionale che m'impegna moltissimo, di mente ed al computer, gli allievi sono dissuasi dall'inviarmi i testi in lettura, tutto avviene in biblioteca, e ora nel teatrino. L'elaborato viene letto ad alta voce e sottoposto alle osservazioni di tutti noi, si legge e si rilegge, spesso si corregge e infine l'ultima parola spetta a me. I testi vengono modificati e rielaborati secondo le indicazioni e solo quando sono pronti vengono archiviati nei file del mio computer. Credevo d'averlo ideato io, questo metodo, squisitamente laboratoriale e coinvolgente, poi una signora del corso mi ha segnalato che in rete ne aveva sentito parlare e si chiama "editing corale". Quello che non esiste, ritengo, è un corso simile al nostro, della stessa lunghezza di mesi e a titolo gratuito in nessuna altro comune d'Italia. Il corso si propone nell'arco di quattro mesi, di far sì che gli allievi scrivano un testo teatrale, imparino a recitarlo e a metterlo in scena. In quattro mesi, due volte la settimana. Un'impresa titanica. La fatica sovrumana è confortata da un gran successo di pubblico e risate a non finire che accoglie i nostri saggi su temi quali: Unità d'Italia, Celebrità, Storie di ordinaria follia. La Crociera Perfetta e La Quinta Stagione (quella in cui si realizzano i sogni).

Ho tratto soddisfazioni enormi negli anni; dapprima vi fu il "Giallo in biblioteca", una commedia esilarante scritta e concepita per essere poi rappresentata all'interno della stessa. Idea semplice e geniale che nell'economia dei mezzi permise una straordinaria messa in scena. Ci divertimmo moltissimo e persone dall'aspetto irreprensibile si trasformarono in teppistelli metallari, assassini e prostitute, scoprendo un gusto per il teatro che non li lascerà più.

Da un soggetto di un'anziana signora, tratto da una storia vera - ed autobiografica ma.. con quale respiro! - "Il segreto" altri allievi scrissero una sceneggiatura che poi si tramutò in "corto". Grazie ad un finanziamento del Comune, potemmo girare gli esterni ma soprattutto rifare il look ad un circolo sociale per retrocederlo agli anni cinquanta.

La storia, commovente e profonda insieme, con lievi tocchi d'ironia, si svolge fra il dopoguerra e il tempo presente e il nostro "corto" ha già avuto numerose riprese per il "Giorno della Memoria". Avere anche noi, nel nostro piccolo, potuto portare un contributo e una testimonianza per dimenticare uno dei lati più oscuri della storia dell'uomo, ci ha reso molto fieri.

Tutti noi ricordiamo con un sorriso le ore passate a ingozzare di gelati le due attrici per fare e rifare la stessa scena sul lungomare di Santa Margherita Ligure oppure 
a trovare la giusta atmosfera per la tragica rivelazione di un numero tatuato su un braccio fra una donna e una bimba, in una osteria negli anni cinquanta.

Le soddisfazioni provengono non solo dai saggi di fine anno, ma dai premi, e molti, che gli iscritti ai corsi hanno vinto, in concorsi di teatro, cinema, di racconti, romanzi e poesie. Premi nazionali e internazionali. Si sarà dedotto che se insegno regole, anche se poche, e su queste non transigo, è che la scrittura teatrale è eminentemente tecnica e paradossalmente è più ostico impartirle a chi ha già scritto, di solito racconti, romanzi e poesie, magari ancora nel cassetto, o peggio, pubblicati a proprie spese. Gli scrittori, se poi anche insegnanti, sono incaponiti e saccenti e spesso abbandonano il corso perché non accettano di rifare il proprio testo secondo i canoni teatrali. Battute del dialogo della lunghezza di mezza pagina, altamente descrittive, con parole ricercate e difficili anche da pronunciare e nessuna traccia di sottotesto.

Qualcuno è umile e rifà molte volte, come è normale che sia e apprezza e riparte alla grande. Vincere queste resistenze è arduo ed estenuante. In queste occasioni pretendo che il "decalogo" sia letto e riletto, fino all'ossessione.

Nei primi tre anni il tema era unico, suggerito da qualche funzionario del Comune preposto alla cultura, ad esempio, “Unità d'Italia" o "Celebrità" e gli allievi scrivevano brevi scenette sull'argomento. Il tono doveva essere brillante e alla fine il risultato era sempre convincente poi... un diavoletto mi spinse a cambiare modulo.

Per quanto per me fosse più semplice assemblare diverse scenette e coordinare entrate e uscite, raddoppiare e triplicare i ruoli, avvalendomi di una efficiente aiuto regia da Daniela, postina-poetessa, da me chiamata "donna di lettere", pure volli cambiare. Volli alzare l'asticella, prendendo a prestito l'espressione dal salto in alto, per andare oltre, superare i propri limiti. Dal tema comune, ora scelto da noi tutti, dopo accalorate discussioni, volevamo trarre un testo teatrale completo. Cominciammo con la Crociera perfetta e la Quinta stagione, quella in cui si avverano i sogni.

Come nelle precedenti edizioni ognuno scrive un pezzo, lo legge e lo discutiamo, ma la grande differenza è che non sono brani a sé stanti, bensì parte di un unico testo. Pertanto bisogna seguire la trama concordata in precedenza, con un plot e un sub plot, vale a dire, trama e sottotrama. Discussa anche quella in precedenza, come un filo rosso che percorra tutto il copione. Vi sfido a farlo con un minimo di 20 persone, di ogni età ecc ecc.

Esempio: per la Crociera perfetta la trama principale era rubare la formula segreta ( è vero!!!) delle palle di vetro con la neve che scende, quelle autentiche, brevettata da una famiglia austriaca. Sottotrama: un gatto a bordo, portato clandestinamente da una stonatissima cantante lirica. 
Per la Quinta stagione la devastazione del territorio, segnatamente quello del Monte Parco di Portofino, Patrimonio Nazionale, preda della speculazione dei miliardari, nella verità e nella finzione scenica. Sottotrama: la ricerca di una studentessa del corso estivo internazionale di musica che si svolge proprio a Santa Margherita, del proprio padre, un tempo guardia del corpo e ora potente oligarca russo. Ovviamente lei questo non lo sa, possiede un unico indizio: un tatuaggio a forma di delfino. La ricerca diventa un tormentone, poiché via via scopre che tutti i personaggi maschili ne hanno uno, persino l'Abate Beato Celeste della Congregazione di Santa Maria Juana ( Mari..Juana) la cui Casa Madre è alle isole Cayman. Il ruolo dell' Abate scritto e interpretato da una signora alta e imponente.

Situazione atta a creare spassosi equivoci a non finire, secondo la tradizione del teatro e anche delle sit-com, cui devo, spesso obtorto collo, fare riferimento.

Nella stesura del testo collettivo i personaggi scritti da un allievo devono poi essere uniformati come caratterizzazione, con lo stesso personaggio scritto dall'altro, e per fare un esempio, nella Crociera perfetta, la marchesa snob descritta da uno era chissà perché diventata una baronessa volgare in un altro.

Alcune scene o dialoghi assolutamente non funzionano, non sono teatrali e pertanto rammento loro di come scrivendo bisogna immedesimarsi totalmente nel personaggio e di come si debba immaginare la scena. Ad una ragazza che aveva ambientato la sua scena nel bosco del monte, con cinghiali, scoiattoli e trappole in cui far cadere gli speculatori edilizi, ho chiesto come lei visualizzava il bosco mentre lo scriveva e lei $\mathrm{mi}$ ha risposto che lo vedeva proprio così, minuziosamente, cioè come una realtà che possa essere descritta in un film o in un romanzo. Le ho fatto notare che per il teatro si "deve vedere" il nudo palco e su questo proiettare il proprio bosco o il proprio salotto, ridotti all'essenzialità, utilizzando la scenografia verbale. All'interno del dialogo e con l'azione, descrivere così vividamente che siamo di fronte ad un cinghiale ( o al fantasma del padre di Amleto) che il pubblico si guarda attorno e lo vede lui stesso.

Poi una volta che il testo con la mia supervisione è finito, ecco che qui viene il bello, loro stessi devono recitarlo!! Tralasciamo che nel frattempo devo insegnare anche i rudimenti della recitazione, per me il vero insegnamento che ne segue è quello precipuo della drammaturgia, dove anche io non smetto di imparare, poiché è nel metterlo in piedi che si rivelano le pecche di un testo. Anche una scena apparentemente ben scritta e da me dunque approvata, sul palco, non si regge.

L'attore in carne ed ossa e sangue figura tridimensionale sul palco deve interpretare personaggi spesso unidimensionali o al massimo bidimensionali e proferire parole di carta, sovente non sentite, perché si deve scrivere col cuore più che con la mente. Quindi si procede con il bisturi e come per incanto, la scena balza agli occhi viva, 
dinamica, essenziale e necessaria. Nel tagliare il dialogo si monta anche l'azione, che dalle didascalie a volte risulta troppo vaga o troppo descrittiva.

Per la comprensione del pubblico magari si aggiunge qualcosa, un dato, un riferimento, anche ripetuti, che però possano fluire impercettibilmente attraverso il dialogo, e si attinge ancora alla scenografia verbale, benedetta tecnica quando non si hanno mezzi per supporti tecnologici, che peraltro per me spesso snaturano il teatro. Infatti ad ogni difficoltà incontrata gli allievi suggeriscono proiezioni sullo sfondo e gli effetti speciali, mentre io li spingo a usare tecniche e vari stratagemmi, e quando assieme li troviamo, un largo sorriso si dipinge sui nostri volti stanchi. Ogni età apporta il suo contributo, e se i più grandi contribuiscono con esperienza di vita vissuta, nell'ampio ventaglio anagrafico degli autori del copione rivestono grande importanza i più piccoli. I bambini presenti, anche se in minoranza fra gli adulti, di solito un paio, imparano presto, scrivono bene seguendo idee imprevedibili, apportando una ventata di aria fresca, e la loro presenza sul palco arricchisce lo spettacolo.

A chi non ha né scritto né recitato ma ha seguito con passione, si possono dare parti quali "panchina" o "gabbiano" , con brevi e facili battute, e la complicità è assicurata, per non parlare degli effetti comici e del sempre profondo insegnamento che a teatro è tutto permesso, si possono fare parlare i morti, gli animali, e gli oggetti, libertà assoluta, poiché tutto è finto ma nulla è falso.

Mi rendo conto che non ho risposto alla domanda del titolo: Drammaturghi si nasceo si diventa? E ancora una volta cito la drammaturga inglese Caryl Churchill: "Scopo del drammaturgo non è dare risposte, bensì porre domande".

\section{BIBLIOGRAFIA}

Ademollo, A. (1888). I teatri di Roma nel secolo decimosettimo: Memorie sincrone, inedite o non conosciute, di fatti ed artisti teatrali, librettisti, commediografi e musicisti, cronologicamente ordinate per servire alla storia del teatro italiano (No. 12). Forni.

Bernardi, C., \& Susa, C. (Eds.). (2005). Storia essenziale del teatro (Vol. 1). Vita e Pensiero.

Bernardi, C., Cuminetti, B., \& Dalla Palma, S. (Eds.). (2000). I Fuoriscena: esperienze e riflessioni sulla drammaturgia nel sociale (Vol. 28). claudio bernardi.

Bosisio, P. (2007). Tra Goldoni e Strehler: Arlecchino e la commedia dell'arte. Tra Goldoni e Strehler: Arlecchino e la commedia dell'arte, 15-95.

Goldoni, C., \& Vendramin, F. (1885). Carlo Goldoni e il teatro di San Luca a Venezia: carteggio inedito (1755-1765) (No. 719). Fratelli Treves.

Jandelli, C. (2011). Breve storia del divismo cinematografico. Marsilio Editori spa.

Mic, C. (1927). La Commedia dell'arte (p. 211). Verlag nicht ermittelbar. 